\title{
The Artificial Neural Networks (ANN) for Batik Detection Based on Textural Features
}

\author{
Anita Ahmad Kasim ${ }^{1}$, Muhammad Bakri ${ }^{2}$, Anindita Septiarini ${ }^{3}$ \\ \{nita.kasim@gmail.com¹ ${ }^{1}$ embakri@gmail.com², anindita.septarini@gmail.com ${ }^{3}$ \}
}

\begin{abstract}
Departemen of Information Technology, Tadulako University, Palu, Indonesia ${ }^{1}$, Department of Architecture, Tadulako University, Palu, Indonesia ${ }^{2}$, Department of Computer Science, Mulawarman University, Samarinda, Indonesia ${ }^{3}$
\end{abstract}

\begin{abstract}
This study aims to utilize artificial neural networks to distinguish batik motifs and non-batik fabric motifs. Several important steps are needed, namely the process of acquiring batik and non-batik images, pre-transforming batik and non-batik images to gray scale forms, texture feature extraction in gray scale images and detection of motifs using networks artificial nerve. Image acquisition is done by collecting batik and not batik images from several different motifs. Processing data sets is divided into $70 \%$ as training data and $30 \%$ as testing data. Artificial neural network models used in this research use the Backpropagation learning algorithm by comparing the Scaled conjugate gradient algorithm (trainscg) training method and the Levenberg-Marquardt algorithm (trainlm) training method. The results obtained for the accuracy using the Scaled conjugate gradient algorithm (trainscg) training method were higher with an accuracy value of $84.12 \%$, compared to the Levenberg-Marquardt algorithm (trainlm) method by $86.11 \%$
\end{abstract}

Keywords: Artificial Neural Networks (ANN), Batik detection, Textural features

\section{Introduction}

Artificial neural network (ANN) is an information processing system that has the same performance characteristics as human biological neural networks. Artificial neural networks have been developed as a form of generalization of mathematical models of human cognition or human biological nerves [1]. Batik has diverse motifs throughout Indonesia. Every region in Indonesia has a batik motif that is tailored to the philosophy of life of each community. Javanese batik is unique in terms of texture, color and shape. Javanese batik can be identified through understanding and knowledge of the motifs and types of batik. Batik can be distinguished based on manufacturing techniques consisting of written batik, batik printing and a combination of writing and printing. The uniqueness of batik comes from the production process known as "mbatik" and the motifs and values contained in each batik motif. Etymologically batik has the meaning of the suffix "tick", in the word "batik" comes from the word drip or drip. The word "tick" can be interpreted as "small", so it can be said "mbatik" is 
to write or draw all complicated / small[2]. In ancient languages it was called fiber, and in ngoko it was called "write" or write with wax [3]. The 'mbatik' process, which is to melt wax and use canting as a means of making batik motifs on fabric. Batik printing is made using plates with batik motifs and printed on fabric. While the combined types of batik are made by combining the two techniques. The problem in this research is the difficulty in distinguishing batik motifs from non-batik cloth motifs for people who do not understand batik motifs. The classification of batik motifs can be done by processing digital images of batik motifs using artificial neural network methods for the classification of batik image motifs. This study aims to utilize artificial neural networks to distinguish batik motifs and non-batik fabric motifs. Artificial neural networks have the ability to model the way of human thinking in distinguishing various objects. This paper is made in five parts, namely introduction, literature review, material and proposed methods, results and discussion and conclusions.

\section{Previous Research}

Variations in batik patterns are very diverse resulting in the start of the development of various studies in the field of batik pattern recognition[4-8]. In pattern recognition, a clear classification rule is needed to recognize the pattern well. Data classification is needed to identify potential characteristics in the $\mathrm{N}$ set of objects contained in the database. These are categorized into several different groups. This study aims to obtain classification rules using the fuzzy decision tree. The research began by obtaining batik image data (pre-processing), enhancement and segmentation (process), feature extraction including entropy, energy, contrast and homogeneity. Next, calculate the value of information acquisition and build a decision tree and finally extract the fuzzy classification rules of batik patterns. Extract classification rules that can classify batik patterns and batik patterns instead[9]. Another method that has been used is the Support Vector machine[10]. The selection of artificial neural networks is done because artificial neural networks have characteristic patterns of connections between neurons that are able to distinguish one object from another. There is a training or learning process to determine the connection weights on the artificial neural network architecture and has an activation function on the artificial neural network architecture. Artificial neural networks have a variety of methods of coaching that can be used to train data so that they can detect different objects. The commonly used training algorithm is the back propagation method[1]. Batik motifs have several characteristics that are used to describe the name. Some batik motifs have clear edges with high contrast values while some batik motifs have vague edges with low contrast values. Meanwhile, the size of the edge of the motif there are thick batik motifs and thin motif edges. Each batik is marked with a main ornament whose size consists of the size of a small ornament and the size of a large ornament. The results obtained by batik ornaments with large size and high contrast produce thick edges and are easy to recognize while batik ornaments with small sizes with low contrast will be difficult to recognize. Two batik images that have high or low contrast with a small image size will produce complex edges [4,11]. Feature extraction and representation is one of the important processes in pattern recognition processing. The feature extraction process for batik images is carried out by transforming batik images into gray scale images. After obtaining an image with gray scale, quantization is performed to obtain a co-occurrence matrix[12]. The cooccurrence matrix that has been obtained is normalized and the statistical feature values are 
obtained based on the co-occurrence matrix. The process of extracting texture features with GLCM produces texture features such as contrast, energy, homogeneity, and correlation [13].

\section{Material And Proposed Methods}

The data used in this study are data on batik images and non-batik images. A total of 117 batik image data consisting of 76 batik images and 43 non batik images. The image is obtained by direct acquisition using a camera with rarely about $90 \mathrm{~cm}$. Examples of batik and non-batik images can be seen in Figure 1

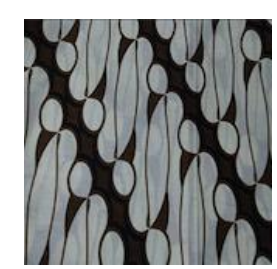

a. batik

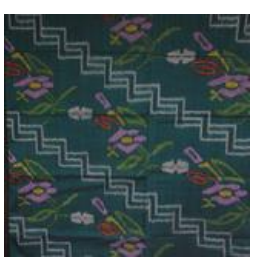

b. non batik

Fig. 1. Image sample of batik and non batik

Artificial neural network method with backpropagation algorithm as follows [1]:

1. Initialize weights with random value. Set learning rate $\alpha$ as $0<\alpha<=1$

2. If the conditions stop not fulfilling, then do Steps 3 to 9.

3. For each pair of training, do steps 4 through 9

Feedforward

4. Each input neuron $\left(x_{i}=1,2, \ldots, n\right)$ receive input and spread the signal to all hidden layer neurons

5. Each unit in hidden layer $\left(z_{j}=1,2, \ldots, p\right)$ add up the weight of the input signal using the Equation 1.

$$
z_{i n_{j}}=V_{0 j}+\sum_{i=1}^{p} x_{i} v_{i j}
$$

then the output value is calculated using the activation function used by the Equation 2.

$$
z_{j}=f\left(z_{i n_{j}}\right)
$$

and send this signal to all the unit in the ouput layer

6. Each unit in output layer $\left(y_{k}, k=1,2, \ldots, m\right)$ add up the weight of the output signal, using the Equation 3.

$$
y_{i n_{k}}=w_{0 k}+\sum_{j=1}^{p} z_{j} w_{j k}
$$

Calculate the output signal using the activation function in the Equation 4.

$$
y_{k}=f\left(y_{i n_{k}}\right)
$$

\section{Backpropagation error}

7. Each output of neuron $\left(y_{k}, k=1,2, \ldots, m\right)$ receive the corresponding target pattern using the input pattern and then calculate the error information using the Equation 5. 


$$
\delta_{k}=\left(t_{k}-y_{k}\right) f^{\prime}\left(y_{i n_{k}}\right)
$$

Calculate the weight correction using the Equation 6.

$$
\Delta w_{j k}=\alpha \delta_{k} z_{j}
$$

Calculate the correction to bias using the Equation 7.

And send these signals to the previous layer

$$
\Delta w_{0 k}=\alpha \delta_{k}
$$

8. Each hidden neuron $\left(z_{j}, j=1,2, \ldots, p\right)$ delta input is derived from neurons in the layer above it using Equation 8.

$$
\delta_{i n_{j}}=\sum_{k=1}^{m} \delta_{k} w_{j k}
$$

Multiply by the derivation of the activation function to find errors using Equations 9 .

$$
\delta_{j}=\delta_{i n_{j}} f^{\prime}\left(z_{i n_{j}}\right)
$$

And calculate the weight of the correction using the Equation 10.

$$
\Delta v_{i j}=\alpha \delta_{j} x_{i}
$$

And calculate the correction bias using the Equation 11.

Update weights and biases

$$
\Delta v_{0 j}=\alpha \delta_{j}
$$

9. Each neuron output value $y_{k}, k=1,2, \ldots, m$ update the bias and weight $\mathrm{j}=0,1, \ldots, \mathrm{p}$ using Equation 12

$$
w_{j k}(\text { new })=w_{j k} \text { old }+\Delta w_{j k}
$$

Each neuron hidden $z_{j}=1,2, \ldots, p$ update the bias and weight $\mathrm{i}=0,1, \ldots, \mathrm{n}$ using Equation 13.

Test the stopping condition

$$
v_{i j}(n e w)=v_{i j}(\text { old })+\Delta w_{i j}
$$

If $\delta_{k}<$ value error initial set then Stop Training

Artificial neural networks especially backpropagation training methods have different training methods. Each backpropagation training method has different computations and different storage needs, and none of the backpropagation training methods can be suitable for all problems. Each problem resolution will use a backpropagation training method that suits the needs of the problem. This study will compare the use of the Scaled conjugate gradient algorithm method and the Levenberg-Marquardt algorithm method. Scaled Conjugate algorithm is a supervised learning algorithm and works for feed forward neural networks. Formulation of Scaled Conjugate algorithm is given as Formulai 14.

$$
S_{k}=\frac{E^{I}\left(w_{k}+\sigma_{k} \cdot P_{k}\right)-E^{I}\left(w_{k}\right)}{\sigma_{k}}+\lambda_{k} \cdot P_{k}
$$

SCG has two initial value parameters $\lambda_{k}$ and $\sigma_{k}$. Value $E\left(w_{k+1}\right) \leq E\left(w_{k}\right)$ is a small number that is used to rectify the special case of converging to a function value of exactly zero. The Levenberg-Marquardt (LM) algorithm is the most widely used optimization algorithm. Its performance is far better than simple gradient descent and other conjugate 
gradient methods in a wide variety of problems. Formulation of Levenberg Marquardt Algorithm is given as Formulai 15.

$$
w_{i+1}=w_{i}-(H+\lambda I)^{-1} d
$$

Where $\lambda$ is blending factor and I is identity matrix. As $\lambda$ gets small the rule approaches the quadratic approximation update rule above. If $\lambda$ is large, the rule approaches as Formulai 16.

$$
w_{i+1}=w_{i}-\frac{1}{\lambda} d
$$

Steps taken to detect batik or non-batik motifs are the process of acquiring batik and nonbatik images, pre-transformation of batik and non-batik images to gray scale forms, texture feature extraction in gray scale images and detection of motifs using artificial neural networks. The method of producing batik image detection with ANN can be shown in Figure 2.

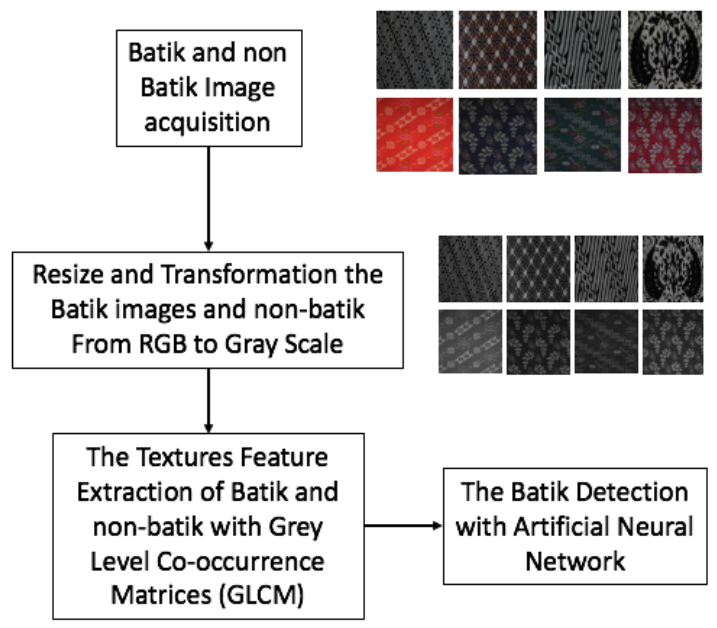

Fig. 2. Metode yang diusulkan.

Image acquisition is done by collecting batik and not batik images from several different motifs. Every batik image is saved in a file with an image format .jpg. The preprocessing process is done by resizing the image to $256 \times 256$.

The method used for texture feature extraction is Gray Level Co-occurrence Matrices (GLCM). GLCM is one method for obtaining second order statistical features by calculating the probability of a neighborhood relationship between two pixels at a certain distance and orientation. The GLCM process approach works by forming an image data cohesion matrix, followed by determining the characteristics as a function of the intermediate matrix. Cooccurrence means a joint event, i.e. the number of occurrences of one level of neighboring pixel value with another level of pixel value within a certain distance (d) and angular orientation $(\theta)$. Distance is expressed in pixels and orientation is expressed in degrees. The orientation is formed in four angular directions with $45^{\circ}$ angle intervals, namely $0{ }^{\circ}, 45^{\circ}, 90$ ${ }^{\circ}$, and $135^{\circ}$. Four features that will be extracted in this study are based on the texture features proposed by the Angular Second Moment (ASM), contrast, correlation and Inverse Different Moment (IDM)[14]. Artificial neural networks model the way neural networks work in 
humans who are able to group data and carry out inference tasks on data. Artificial neural networks can act like humans in understanding, thinking, remembering, inferring, and knowing. Artificial neural networks are very powerful instruments for the classification of data and conclusions [15]. The practical concept of ANN to detect batik images is by processing the characteristics of batik image patterns and training them to be able to detect based on batik motifs and non-batik motifs. The neural network consists of a number of processing elements called neurons, units, cells or nodes [16]. The model of a neuron in artificial neural networks is shown in Figure 3.

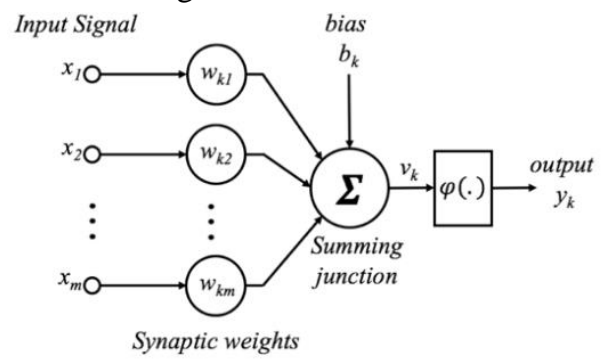

Fig. 3. Neuron Model.

Figure 3 shows a neuron model consisting of three basic elements, namely:

- A group of synapse or connecting links that have different weights

- An addition unit that will add up the input signals that have been multiplied by the weights

- An activation function that determines whether an input signal will be forwarded to other neuron.

Each interconnected neuron can be interpreted as a form of communication and each communication is related to weight. Weights represent the information used to solve problems. These neurons accumulate in layers called neurons. The layers making up artificial neural networks are divided into three, namely:

- Neurons contained in the input layer receive input patterns from outside which describe the problem (Input layer)

- Neurons in the hidden layer receive the input layer pattern (hidden layer)

- Neurons in the output layer are the solution or the result of artificial neural networks (output layer)

Artificial neural network models used in research use the Back propagation learning algorithm by comparing the training method of the Scaled conjugate gradient algorithm (trainscg) and the Levenberg-Marquardt algorithm (trainlm). Levenberg-Marquardt algorithm (trainlm) is one of the fastest training algorithms for networks of moderate size. This method has a feature to reduce memory used when the size of training data is large. In addition there is the Scaled conjugate gradient algorithm (trainscg). The dataset processed in this study were 119 images consisting of 76 batik images and 43 non-batik images. Sixteen texture features are used, namely Angular Second Moment (ASM), contrast, correlation and Inverse Different Moment (IDM) with $45^{\circ}$ angle intervals, namely $0^{\circ}, 45^{\circ}, 90^{\circ}$ and $135^{\circ}$. Processing data sets is divided into 70 percent as training data and 30 percent as test data. The testing of the batik motif classification model conducted in this study includes performance appraisal. Performance assessment, namely testing the ability of pattern classification models in classifying data that has errors in classification. 


\section{Result And Discussion}

This study conducted image detection to determine whether the image of batik or not batik. There are two learning methods to be used namely the Scaled conjugate gradient algorithm method and the Levenberg-Marquardt algorithm method. The training method used will train the entered image data and group them into batik image class or non-batik image. The results obtained there are batik images detected as batik images, there are non-batik images detected as non-batik images, there are batik images but detected as not and there are non-batik images but detected as batik. The correct detection accuracy of batik and non-batik images is obtained by adding up the values that can be detected correctly divided by the total amount of image data. The results of batik image detection can be shown in Table 1

Table 1. Result of batik and non batik detection.

\begin{tabular}{lccc}
\hline \multirow{2}{*}{ Experiment } & \multicolumn{2}{c}{ Output Class } \\
\cline { 3 - 4 } & Non Batik & True & False \\
\hline \multirow{2}{*}{ Target Class } & Batik & False & True \\
\hline
\end{tabular}

This study will compare the use of the Scaled conjugate gradient algorithm method and the Levenberg-Marquardt algorithm method. The number of neurons in each was determined based on the number of inputs (the number of texture features) and the number of outputs (batik and non-batik). For the number of neurons in the hidden layer the modified number. Experiments carried out as many as 14 experiments with 2 training functions and a combination of the number of neurons in each layer. The results of the batik detection model experiment with the Scale Conjugated Gradient algorithm training method are shown in Table 2.

Table 2. Results of batik detection model experiments with Scale Conjugated Gradient training methods.

\begin{tabular}{cccccrr}
\hline \multirow{2}{*}{ Experiment } & \multirow{2}{*}{$\begin{array}{c}\text { Training } \\
\text { Function }\end{array}$} & $\begin{array}{c}\text { Input } \\
\text { Layer }\end{array}$ & $\begin{array}{c}\text { Hidden } \\
\text { Layer }\end{array}$ & $\begin{array}{c}\text { Output } \\
\text { Layer }\end{array}$ & Accuracy & $\begin{array}{c}\text { Error } \\
\text { Rate }\end{array}$ \\
\cline { 3 - 6 } & & 16 & 2 & 1 & 100 & 0 \\
2 & 16 & 4 & 1 & 100 & 0 \\
3 & 16 & 6 & 1 & 100 & 0 \\
4 & Train SCG & 16 & 8 & 1 & 83.3 & 16.7 \\
5 & 16 & 10 & 1 & 75 & 25 \\
6 & 16 & 12 & 1 & 63.88 & 36.12 \\
7 & 16 & 16 & 1 & 66.66 & 33.34 \\
\hline & & & & 84.12 & 15.88 \\
\hline
\end{tabular}

Experiments 1 through 7 are carried out by combining the number of neuorons in the hidden layer. The number of neurons in the hidden layer ranges from 2 to 16 with an average 
accuracy of all experiments of 84.12 percent and an error rate of 15.88 percent. The best accuracy is in experiments with using a number of neurons 2, 4 and 6 of 100 percents. The lowest accuracy in p uses as many as 12 neurons. The results of the batik detection model experiment with the Levenberg-Marquardt algorithm training method are shown in Table 2. Experiments 8 through Experiment 14 were carried out by combining the number of neuorons in the hidden layer. The number of neurons in the hidden layer ranges from 2 to 16 with an average accuracy of all experiments of 86.11 percent and an error rate of 13.89 percent. The best accuracy is in experiments using the number of neurons 8, 12 and 16 of 100 percent. The lowest accuracy is using 10 neurons. Results of the batik detection model experiments with the Levenberg-Marquardt algorithm training method are shown in Table 3.

Table 3. Results of the batik detection model experiments with the Levenberg-Marquardt algorithm training method.

\begin{tabular}{cccccrr}
\hline \multirow{2}{*}{ Experiment } & \multirow{2}{*}{$\begin{array}{c}\text { Training } \\
\text { Function }\end{array}$} & $\begin{array}{c}\text { Input } \\
\text { Layer }\end{array}$ & $\begin{array}{c}\text { Hidden } \\
\text { Layer }\end{array}$ & $\begin{array}{c}\text { Output } \\
\text { Layer }\end{array}$ & Accuracy & $\begin{array}{c}\text { Error } \\
\text { Rate }\end{array}$ \\
\cline { 3 - 6 } & & 16 & 2 & 1 & 94.44 & 5.56 \\
9 & 16 & 4 & 1 & 66.66 & 33.34 \\
10 & 16 & 6 & 1 & 77.77 & 22.23 \\
11 & TrainLM & 16 & 8 & 1 & 100 & 0 \\
12 & 16 & 10 & 1 & 63.88 & 36.12 \\
13 & 16 & 12 & 1 & 100 & 0 \\
14 & 16 & 16 & 1 & 100 & 0 \\
\hline
\end{tabular}

\section{Conclusion}

The conclusion in this study is the detection model of batik images or not batik images built by modeling artificial neural networks. The dataset were 119 images consisting of 76 batik images and 43 non-batik images. Sixteen texture features are used, namely Angular Second Moment (ASM), contrast, correlation and Inverse Different Moment (IDM) with $45^{\circ}$ angle intervals, namely $0^{\circ}, 45^{\circ}, 90^{\circ}$ and $135^{\circ}$. The average accuracy obtained by using the Scale Conjugated Gradient training methods training method is 84.12 Percent and the average accuracy for the Levenberg-Marquardt algorithm training method is 86.11 percent. There is a difference of about 2 percent accuracy for the batik detection model with artificial neural networks.

Acknowledgment. This work supported and funding by RISTEKDIKTI Indonesia in the scheme "Penelitian Dasar" in 2019 with Grant Number 351.r/UN28.2/PL/2019 


\section{References}

[1] Fausett, L.: Fundamental of Neural Networks: Architecture, Algorithm and Applications. New Jersey: Prentice Hall (1993)

[2] Sugiyem: Makna Filosofi Batik. WUNY Lemb. Pengabdi. Kpd. Masy. Univ. Negeri Yogyakarta, Vol. X, pp. 1-10 (2008)

[3] Haake, A.: The Role of Symmetry in Javanese batik Patterns. Comput. Math. Appl. Appl., Vol. 17, No. 4, pp. 815-826 (1989)

[4] Moertini, V. S. and Sitohang, B.: Algorithms of Clustering and Classifying Batik Images Based on Color, Contrast and Motif. Vol. 37, No. 2, pp. 141-160 (2005)

[5] Moertini, V. S.: Towards classifying classical batik images (2005)

[6] Arisandi, B., Suciati, N., and Yudhi, A.: Pengenalan Motif Batik Dengan Rotated Wavelet Filter Dan Neural Network. Surabaya (2011)

[7] Minarno, A. E., Munarko, Y., Kurniawardhani, A., Bimantoro, F. and Suciati, N.: Texture Feature Extraction Using Co-Occurrence Matrices of Sub-Band Image For Batik Image Classification. In 2nd International Conference on Information and Communication Technology (ICoICT) Texture. pp. 249-254 (2014)

[8] Aditya, C. S. K., Hani'ah, M., Bintana,R. R., and Suciati, N.: Batik Classification Using Neural Network With Level Co-Occurence Matrix And Statistical Color Feature Extraction. In International Conference on Information, Communication Technology and System (ICTS). pp. 163-168 (2015)

[9] Kasim, A. A. and R. Wardoyo, R.: Batik Image Classification Rule Extraction using Fuzzy Decision Tree. In Information Systems International Conference (ISICO). No. December, pp. 2-4 (2013)

[10] Nuraedah, Bakri, M. and A. A. Kasim, A. A.: Quadratic support vector machine for the bomba traditional textile motif classification. Indones. J. Electr. Eng. Comput. Sci., Vol. 11, No. 3, pp. 1004 1014 (2018)

[11] Kasim, A. A. and Harjoko, A. Klasifikasi Citra Batik Menggunakan Jaringan Syaraf Tiruan Berdasarkan Gray Level Co- Occurrence Matrices ( GLCM ). In Seminar Nasional Aplikasi Teknologi Informasi (SNATI). pp. 7-13 (2014)

[12] Rullist, Y. Irawan, B. and Osmond, A. B.: Batik's Pattern Identification Through Feature Extraction Method, Gray Level Co - Occurrence Matrix (Glcm), Based On Android. e-Proceeding Eng. Vol. 2, No. 2, pp. 3684-3692 (2015)

[13] Kasim, A. A., Wardoyo, R. and Harjoko, A. Fuzzy C Means for Image Batik Clustering Based on Spatial Features. Int. J. Comput. Appl. Vol. 117, No. 2, pp. 1-4, (2015)

[14] Haralick, R., Shanmugan, K. and Dinstein, I. Textural features for image classification. IEEE Trans. Syst. Man Cybern. Vol. 3, pp. 610-621 (1973)

[15] Malmgren, H.:Artificial Neural Networks in Medicine and Biology. In Opening lecture at the ANNIMAB-1 conference, Göteborg. Vol. 1, No. 21, pp. 1-21 (2000)

[16] Haykin, S. S.: Neural Networks and Learning Machines, 3rd Edition (2009)

[17] Webb, A. R. and Copsey, K. D.: Statistical Pattern Recognition, Third. A John Wiley \& Sons, Ltd, Publication (2011) 